\title{
Modulation of host lipid membrane elasticity and dipole potential induced by bacterial membrane vesicles
}

\author{
Ashutosh Prince ${ }^{1}$, Anuj Tiwari ${ }^{1 \dagger}$, Titas Mandal ${ }^{1 \dagger}$, Kuldeep Sharma ${ }^{1}$, Nikhil Kanik ${ }^{1}$, Deepak Kumar \\ Sinha², Mohammed Saleem ${ }^{3,4 *}$ \\ ${ }^{1}$ Department of Life Sciences, National Institute of Technology, Rourkela, India. \\ ${ }^{2}$ Department of Biological Chemistry, Indian Association for the Cultivation of Sciences, Kolkata, \\ India. \\ ${ }^{3}$ School of Biological Sciences, National Institute of Science Education and Research, \\ Bhubaneswar, India. \\ ${ }^{4}$ Homi Bhabha National Institute, Mumbai, India. \\ * Mohammed Saleem. \\ $\dagger$ Equal authorship \\ Email: saleem@niser.ac.in

\section{Keywords}

Bacterial Membrane Vesicles; Dipole Potential; Anisotropy; Compressibility Modulus.

\section{Author Contributions}

AP and MS conceived the idea and designed the research. AP, AT and KS carried out the characterization of bacterial MVs. DKS helped with MV characterization. AP and NK performed fluorimetry experiments. AP and AT performed labelling of bacterial MVs and imaging experiments. AP and TM performed monolayer experiments. AP, AT, TM and MS analysed the data. AP, AT, TM and MS wrote the manuscript. 


\begin{abstract}
Bacterial membrane vesicles (MVs) facilitate long-distance delivery of virulence factors crucial for pathogenicity. The entry and trafficking mechanisms of virulence factors inside host cells is recently emerging, however, if bacterial MVs modulate the physicochemical properties of the host lipid membrane remains unknown. Here we reconstitute the interaction of bacterial MV with host cell lipid membranes and quantitatively show that bacterial MV interaction increases the fluidity, dipole potential and elasticity of a biologically relevant multi-component host membrane. The presence of cylindrical lipids such as phosphatidylcholine and phosphatidylinositol and a moderate acyl chain length of $\mathrm{C} 16$ helps the MV interaction. While significant binding of bacterial MVs to the raft-like lipid membranes with phase separated regions of the membrane was observed, however, MVs have a preference for binding to the liquid disordered regions of the membrane. Further, the elevated levels of cholesterol tend to hinder the interaction of bacterial MVs. We further quantify the change in excess Gibbs free energy of mixing of bacterial MVs with host lipid membranes driving the modulation of host membrane parameters. The findings may have significant implications on the regulation of host machineries by pathogen through manipulation of host membrane properties.
\end{abstract}

\title{
Significance Statement
}

Bacterial membrane vesicles (MVs) act as the long-distance delivery tools for virulence factor and thus, directly implicated in host-pathogen interactions and pathogenicity. While the mechanisms of virulence transfer is only recently emerging, however, the interaction of MVs the host cell membrane remains largely unexplored. Whether the MVs interaction can locally modulate the host lipid membrane physicochemical properties (such as fluidity, dipole potential and elasticity) remains unknown. Here, we quantitatively investigate the lipid specificity of $E$. Coli MV interaction and this results in increase in the fluidity, dipole potential and in-plane elasticity of a biologically relevant multi-component host membrane. The findings could be important for numerous cell-signaling processes as well as downstream events involving membrane-membrane fusion during process of phagosome maturation. 


\section{Introduction}

The secretion of membrane vesicles (MVs) from the bacterial cells is recognized as a generalized phenomenon observed in many bacteria $(1,2)$. Bacterial MVs are known to facilitate contact-free inter-species communication, nutrient acquisition, enhanced survival against immune response and host-pathogen interaction(3). Initially known to be only secreted by controlled blebbing of Gram negative bacteria, emerging evidence supports the existence of different types of Bacterial MVs, such as - Bacterial MVs, Outer-inner membrane vesicles (OIBMVs) and Explosive outer membrane vesicles (EOBMVs) all of which are involved in transport of virulence factors(4). Typically, bacterial MVs are nano-sized spherical membrane compartments spanning 20-300 nm in diameter derived from the outer membrane lipid bilayer and the periplasm of the bacteria(3). Biochemically, bacterial MVs are known to carry lipopolysaccharides (LPS), phospholipids, peptidoglycans, outer membrane proteins (OMPs), nucleic acids, signaling molecules and periplasmic, cytoplasmic and membrane-bound proteins(5). The degree of bacterial MV secretion has also been correlated to the pathogenicity and virulence of bacteria(6). Interestingly, in addition to delivering a selection of virulence factors and immunomodulatory molecules directly into host cells during infection, bacterial MVs are also known to act as decoy antigen and divert the host immune system away from the bacterial cell (7). There is a considerable focus on the understanding of the virulence factors of the MV cargo and modes of bacterial MV entry inside host cells. Owing to a wide range of size of bacterial MVs, different pathways such as macropinocytosis, clathrin-dependent and caveolin or lipid raft mediated endocytosis have been implicated in the internalization processes of different Bacterial MVs (8). Further, direct membrane fusion with host cell membranes, preferentially at lipid raft domains, has also been reported to be a potential mechanism of entry of bacterial MVs (9). Similarly, fusion of bacterial MVs extracted from L. pneumophila with host model membranes suggested the faster time scales of fusion events (few seconds), which is also temperature-dependent (10).

A crucial aspect that remains unexplored is if/how MV fusion modulates the physicochemical parameters of host cell membranes (i.e., fluidity, dipole potential and in-plane elasticity)? Irrespective of the kind of bacterial MVs, mode of entry of bacterial MVs, insights on membrane interfacial interaction of bacterial MVs is crucial as the local lipid reorganization and remodeling of the host cell membrane is a prerequisite for MV entry. It is proposed that the MV fusion may result in changes in the properties of the host cell membrane(10), however, there is no experimental evidence in support of this idea. The lipid specificity, quantitative changes in physicochemical parameters (e.g., fluidity, dipole potential and in-plane elasticity) of host membrane triggered by bacterial MVs and the thermodynamics of their interaction has never been explored to best of our knowledge.

Using in vitro reconstitution, fluorescence microscopy, potentiometric dye-based fluorescence anisotropy and dipole potential measurements and surface pressure-area isotherms, we show that MV interaction increases the fluidity, dipole potential and in-plane elasticity of the lipid membranes mimicking the host cell membrane. We also report the role of host membrane lipid head-group specificity, acyl chain length and phase separated boundaries on the interfacial interaction of bacterial MVs. The presence of lipids such as 1,2-dioleoyl-sn-glycero-3-phosphocholine (DOPC), 2-dioleoyl-sn-glycero-3-phospho-(1'-rac-glycerol) (DOPG) and phosphatidylinositol (PI) and an acyl chain length of $\mathrm{C} 16$ are significant for the fusion of the bacterial MVs. Furthermore, the bacterial MVs have a preference for liquid disordered regions of the lipid membrane, whereas, the elevated levels of cholesterol in raft-like lipid membranes with phase separated regions tend to hinder the interaction. The findings are further supported by the investigation of the thermodynamic parameters of MV interaction with host lipid membrane, revealing the change in Gibbs excess free energy of mixing of MV components with biologically relevant host membrane lipid membrane conditions. These findings show that bacterial membrane vesicles can effectively modulate the 
physicochemical properties of the host membrane that might eventually have consequences for membrane associated signaling during host-pathogen interactions.

\section{Results}

\section{Bacterial membrane vesicle (MV) fusion increases the fluidity and dipole potential of host lipid membrane}

Bacterial membrane vesicles have been reported to enter host cells via multiple routes such as endocytosis, micropinocytosis and fusion in different cell types (11). Here, we sought to elucidate the lipid specificity and modulation of host membrane properties by bacterial MV interaction which remains central to all routes of entry yet remains unexplored. We used E. coli as a model Gram negative bacterium for the isolation of bacterial MVs and set out to systematically investigate the interaction of bacterial MVs with host membranes (Fig. 1A). Bacterial MVs were isolated by means of chemically induced vesiculation (as described in methods), which in comparison to the usual ultracentrifugation-based isolation, yield a relatively homogenous size of MVs. The characterization of the size heterogeneity of bacterial MVs was carried out by electron microscopy, fluorescencebased pixel size estimate and dynamic light scattering. Electron microscopy of MVs showed that MVs isolated by ultracentrifugation-based method had a broad size range of $\sim 50-200 \mathrm{~nm}$, unlike, chemically induced vesiculation method that yield MVs with diameter of $\sim 50-60 \mathrm{~nm}$ (Fig.1B-1D). The same was found to be $\sim 150 \mathrm{~nm}$ by dynamic light scattering (Fig. 1E). Further, we labelled bacterial MVs with fluorescent lipophilic dyes - such as 3,3'-dioctadecyloxacarbocyanine perchlorate $(\mathrm{DiO})$ and Nile red, to allow visualization of OMV interaction/fusion by confocal microscopy as they are known to fluoresces only under lipid-rich environment (Fig. 1F-H) (12). We then confirmed the internalization of bacterial MVs by human macrophages, as also reported earlier $(10,13)$. The labelled bacterial MVs (devoid of any free unbound dye) were incubated with the macrophage cells in cell media for a period of about 2.5 hours to monitor the changes in the bacterial MV fluorescence arising in the cell media. Measurement of fluorescence intensity of a fixed volume of isolated cell media every $30^{\text {th }}$ minute revealed a gradual yet linear decrease in the fluorescence arising from the residual labelled MV (Fig. 1I). This methodology also provides an indirect measure of the kinetics of the bacterial MV fusion with the macrophage cells. Fluorescence bleaching can be ruled out as no significant change in fluorescence of the cell media containing MV was observed in the absence of macrophage cells (Fig. 1I).

Previous investigations have reported that Bacterial MVs can not only associate with the host cell surfaces but also incorporate with different phospholipid liposomes in vitro (10). Irrespective of the kind of bacterial MV, the interaction with the host lipid membrane takes place between the outer membrane of the bacterial MVs and the host membrane. To this end, we reconstituted giant unilamellar vesicles (GUVs) comprising of the outer leaflet lipids of a eukaryotic cells (i.e., DOPC 35 mole \%, DOPG 15 mole \%, Liver PI 5 mole \%, DOPE 5 mole \%, Brain SM $20 \%$, and Cholesterol $30 \%$ ), in order to mimic the host cell membrane (14). To visualize the interaction of bacterial MVs, we labeled GUVs with Rhodamine-PE (emission wavelength, $576 \mathrm{~nm}$ ) and bacterial MVs with DiO (emission wavelength, $501 \mathrm{~nm}$ ). Confocal microscopy revealed the binding of bacterial MVs with the outer leaflet model membranes (OLMM) (Fig. 2A). The kinetics of binding of bacterial MVs to the host membrane comprising outer leaflet lipids appeared to be fast as reported earlier in different cell lines (15). We next sought to investigate whether the interaction of the bacterial MVs with the host membrane results in changes in the fluidity and dipole potential $\left(\Psi_{\mathrm{d}}\right)$ of the host membrane. The changes in membrane fluidity were quantified by reconstituting a potentiometric styryl membrane probe di-8-ANEPPS (Pyridinium, 4-[2-[6-(dioctylamino)-2-naphthalenyl]ethenyl]-1-(3sulfopropyl)), with membrane vesicles, whose fluorescence anisotropy (a measure of rotational relaxation time) responds to the changes in the dynamics of the membrane environment. The fluorescence anisotropy of the host outer leaflet model membrane was found to decrease by $\sim 9$ $\%$ upon MV interaction suggesting increased fluidization of the host membrane (Fig. 2B). Likewise, the probe di-8-ANEPPS, is known to undergo shift in the fluorescence excitation spectrum in linear 
response to dipole potential changes in the lipid environment, which in turn, is dependent on the electric field in the membrane environment (16-18). The dipole potential originates from the nonrandom arrangement of molecular dipoles (i.e., water, carbonyl-groups and lipid head-groups) at the membrane interface (19). The dipole potential of the host outer leaflet model membrane was found to increase by $\sim 67 \%$ upon the interaction of MV suggesting strong interaction (Fig. $2 \mathrm{C}$ ). Further, the surface zeta potential of the bacterial MVs was found to be $\sim-9 \mathrm{mV}$ and that of the outer leaflet model membrane lipid components was also found to be negative, however, of higher magnitude (- $20 \mathrm{mV}$ to $-25 \mathrm{mV}$ ) as compared to bacterial MVs (Table S1).

\section{Bacterial MV fusion increases the elasticity and facilitates bending of host model membrane}

After identifying the lipid specificity of MV interaction and estimating the accompanied changes in the fluidity and dipole potential of the host membranes, we further sought to quantify the observed changes at a molecular level. Langmuir monolayer surface area-pressure isotherms are a powerful means that enable quantification of the thermodynamic aspects of the MV interaction with the host model membranes and the associated changes in physicochemical properties of the host membranes (19). Further, the variation of compositional parameters mimicking the biological conditions in monolayer experiments allows to dissect the contribution/modulation of hydrophobic interactions, charges, dipole potential during the MV-host membrane interaction.

We first investigated the surface pressure-area $(\pi-A)$ isotherms for the outer leaflet model membrane (OLMM). OLMM being a complex multi-component lipid mixture only showed liquidexpanded $\left(L_{e}\right)$ and -condensed $\left(L_{c}\right)$ behaviour, without any significantly evident 2D phase transition (Fig. 2D). Marked increase in the area per molecule with increasing surface pressure was observed, in the presence of MV in the sub-phase suggesting significant interaction and fusion between the monolayer membrane and bacterial MVs (Fig. 2D). To further corroborate our conclusions, the changes in the host model membrane surface compressional modulus, $\mathrm{Cs}^{-1}$ (i.e., reciprocal of surface compressibility or elasticity, $\mathrm{C}_{\mathrm{s}}$ ), were extracted using the slopes obtained from $(\pi-A)$ isotherms. Compressibility modulus, $\mathrm{C}_{\mathrm{s}}{ }^{-1}$, is related to the change in surface pressure and area per molecule, as defined in equation (1) -

$$
C_{s}^{-1}=-A \frac{d \pi}{d A}
$$

and is known to be very sensitive to subtle changes in lipid structure during lateral interaction and thus, provides molecular insights into lateral packing elasticity of the host membrane (20-22). Upon the fusion of bacterial MVs, the monolayer compressibility modulus was found to have decreased by $\sim 7 \mathrm{mN} / \mathrm{m}$ (Fig. 2E), particularly nearing a surface pressure of $30-35 \mathrm{mN} / \mathrm{m}$ which has been established to possess identical chemical potential and molecular area to that of bilayers in equilibrium (23). Further, the excess Gibbs free energy of mixing of bacterial MVs with OLMM was calculated at certain surface pressures by integrating the excess area over surface pressure, and denoted by the equation (2) (24) -

$$
\Delta \mathrm{G}_{\mathrm{Excess}}=\int_{0}^{\pi}\left[A_{12}-\left(X_{1} A_{1}+X_{2} A_{2}\right)\right] d \pi
$$

where, $A_{12}$ is the mean molecular area occupied by the mixed monolayer in the presence of $\mathrm{MV}$, $A_{1}$ and $A_{2}$ are the mean molecular areas occupied by monolayer and bacterial MVs respectively, and $X_{1}$ and $X_{2}$ are the molar fractions of the monolayer component and bacterial MVs respectively. $\Delta G_{\text {Excess }}$ showed a decrease with increasing surface pressure suggesting the mixing of MV with monolayer lipids to be more favourable till a surface pressure at $10 \mathrm{mN} / \mathrm{m}$ whereas slight increase was observed at $15 \mathrm{mN} / \mathrm{m}$ and $20 \mathrm{mN} / \mathrm{m}$ (Fig. $2 \mathrm{~F}$ ), as the maximum crowding of bacterial MVs approached as reflected in the (Fig. S2). The difference of the collapse pressures $\left(\pi_{c}\right)$ of monolayer 
membranes in the presence and absence of MV depicts the change in bending force $\left(\Delta \mathrm{F}_{\mathrm{b}}\right)$ acting on the monolayer during the adhesion which was calculated using the equation (3):

$$
\Delta F_{b}=M M \cdot M V \pi_{c}-M M \pi_{c}
$$

where, $M M . M V \pi_{c}$ is the collapse pressure of model membrane with bacterial MVs in subphase and $M M \pi_{c}$ being without bacterial MVs. Negative values of $\left(\Delta \mathrm{F}_{\mathrm{b}}\right)$ suggest bending of monolayer (25). The $\left(\Delta \mathrm{F}_{\mathrm{b}}\right)$ was found to be $\sim-1.75 \mathrm{mN} / \mathrm{m}$ suggesting the feasibility of bending upon adhesion of bacterial MVs (Table S2). Highest surface pressure of $20 \mathrm{mN} / \mathrm{m}$ was taken in each case as it is the collapse pressure of MV components monolayer (Fig. S2). In addition, we also examined the $\pi-A$ isotherms of the MV components (i.e., lipopolysaccharides, phospholipids devoid of any proteins) extracted from bacterial MVs (see methods for biphasic solvent extraction). A clear gaseous phase was observed in the beginning followed by $L_{e}$ phase and a short $L_{c}$ phase just before the collapse at around $21 \mathrm{mN} / \mathrm{m}$ (Fig. S2). The compressibility modulus showed a sharp rise till a surface pressure of $2-3 \mathrm{mN} / \mathrm{m}$ from which it became constant at a value of $16 \mathrm{mN} / \mathrm{m}$. Both lower collapse pressure and constant compressibility modulus suggest a significantly rigid behaviour resisting compression (Fig. S2).

\section{Role of host membrane lipid head group and acyl chain length in bacterial MV interaction}

We next questioned if there is any lipid specific preferential interaction of the bacterial MVs with the host cell membrane outer leaflet that majorly contributes towards interaction/fusion of bacterial MVs. To probe this, we reconstituted liposomes composed of the major phospholipids present in the outer leaflet of the host cell membrane i.e., DOPC, DOPG, BSM and Liver PI. We analyzed the binding and colocalization of bacterial MVs with lipid membranes i.e. DOPC, DOPG and Liver PI, to visualize lipid-bacterial MVs interaction (Fig. 3B). Significant binding of bacterial MVs to all the above lipid membranes was observed as evident from the homogenous DiO signal (green) arising from the equatorial plane of giant unilamellar vesicels (GUVs) colocalizing with Rhodamine PE signal (red). After confirming the interaction of bacterial MVs with DOPC, DOPG and Liver PI lipids, we further quantified the modulation of fluidity and dipole potential changes in these lipid membranes. We found that DOPC, DOPG, BSM and Liver PI membranes, all undergo significant changes in the membrane fluidity upon the interaction of bacterial MVs. Interestingly, the fluorescence anisotropy of the dye was found to decrease in case of DOPC, DOPG and Liver PI by $\sim 66 \%, 45 \%$ and $45 \%$ respectively, suggesting enhanced fluidization (Fig. 3C-3E). On the contrary, the membrane composed of BSM showed an increase in the fluorescence anisotropy of the dye by $7 \%$, suggesting the membrane becoming more rigid (Fig. S3). We then investigated the role of lipid head group on the MV induced dipole potential changes in the host membrane. We observed that membranes composed of DOPC, DOPG and Liver PI undergo significant changes in their dipole potential (i.e., $21 \%, 15 \%$ and $9 \%$ respectively) (Fig 3F-3H). On the contrary, membranes composed of BSM did not undergo any significant change in the measured dipole potential (Fig. S3). The lipid acyl chain length is known to determine the membrane bilayer thickness as well as contributes to the degree of fluidity of the membranes (26). Therefore, we then sought to investigate the role of lipid acyl chain (i.e., C12, C14, C16, C18) of host membrane in the MV mediated changes in the overall host membrane fluidity and dipole potential. The overall fluorescence anisotropy of the dye was found to decrease by $30 \%$ in DPPC suggesting increased fluidization (Fig. 4C), and increase by $~ 28 \%, \sim 43 \%$ and $\sim 27 \%$ in DLPC, DMPC and DSPC respectively, suggesting decrease in fluidity (Fig. 4A, 4B and 4D). The contrasting observation is noteworthy, as it suggests, that MV interaction seems to enhance the fluidity of a relatively less fluid membrane (such as DPPC, C16) and decrease the fluidity of relatively more fluid membranes (such as C12,14). The observed fluctuations in the fluorescence anisotropy with increasing concentrations of the MV, could hint at a dynamic interaction during the crowding/interaction of the MV. Further, the dipole potential was found to increase in all cases (DMPC, DPPC and DSPC) but decrease in DLPC membranes upon MV interaction (Fig. 4E-4H). In case of DMPC (Figure 4F) and 
DSPC (Figure $4 \mathrm{H}$ ) in highest concentration the dipole potential was increased to $\sim 30 \%$ and $\sim 15 \%$ respectively, as compared to control which confirms interaction of bacterial MVs with DMPC and DSPC membrane. Likewise, DPPC membrane shows significant increase in dipole potential upon interaction with MV (Fig. 4G). On the contrary, MV interaction with DLPC membranes resulted in less significant change in the dipole potential (Fig. 4E).

\section{MV fusion prefers disordered regions of the host cell membrane}

Host cell membranes are multi-component lipid assembly that is a non-equilibrium homogenous system close to phase separation. The membrane environment has highly transient phase separating regions called rafts important for cellular signaling. We therefore investigated the effect of phase behavior on the interaction of the bacterial MVs. In order to probe this, we reconstituted liposomes (GUVs and LUVs) made of widely accepted ternary membrane composition (i.e., DOPC:BSM:Cholesterol) and varied the amounts of cholesterol to enable different degrees of liquid-disordered $\left(L_{d}\right)$ and liquid-ordered $\left(L_{0}\right)$ regions. This would mimic the biologically relevant highly dynamic local changes in membrane phase boundaries (27). First, we reconstituted GUVs composed of DOPC/BSM without any cholesterol representing a fully disordered $\left(L_{d}\right)$ phase in membrane to examine how the $L_{d}$ forming membrane condition effects the interaction of MV (liquid disordered; DOPC:BSM:Chol 5:5:0). Uniform adhesion/fusion of bacterial MVs to the fully disordered membrane was observed. We arrived at this conclusion by monitoring all the planes of stacks as well as 3D-stack image of GUV incubated with bacterial MVs (Fig. 5A). The fluorescence anisotropy of the dye increased by $30 \%$ upon interaction with MV in a concentration dependent manner indicating decrease in membrane fluidity (Fig. 5B). Further, the membrane dipole potential was found to linearly decrease upon the interaction of MV (Fig. 5C), all of which hint at binding of bacterial MVs that may allow some lipid mixing (28).

We then modulated the degree of phase separation, which in turn effects the line tension at the phase separating boundaries (29), by reconstituting membranes having $\mathrm{L}_{\mathrm{o}} / \mathrm{L}_{d}$ phase separated regions by changing the cholesterol levels (i.e, DOPC:BSM:Chol, 4:4:2/3:3:4). Interestingly, in case of $L_{o} / L_{d}$ phase separating membranes with moderate levels of cholesterol (DOPC:BSM:Chol, 4:4:2) significant binding of bacterial MVs was observed (Fig. 5D). The fluidity decreased in a similar manner as for the Lo membrane as reflected in the increase fluorescence anisotropy of the dye by $15 \%$ (Fig. 5E). Similarly, the dipole potential was found to linearly decrease upon the interaction of MV (Fig. 5F). Interestingly, in case of phase separating membranes comprising higher amount of cholesterol (DOPC:BSM:Chol, 3:3:4), having a larger $\mathrm{L}_{\circ}$ (liquid ordered) regions the MV binding was found to be relatively weak (Fig. $5 \mathrm{G}$ ). Also, the fluidity was found to decrease as in earlier cases, however, without any change in the dipole potential of the membrane (Fig. $5 \mathrm{H}$ and $5 \mathrm{I}$ ).

\section{Elasticity and free energy changes in host model membrane monolayers induced by bacterial membrane vesicles (MVs)}

We sought to investigate the modulation of membrane elasticity upon MV fusion and mixing with regard to various lipid head-groups, acyl chain length and phase separated membranes. We observed, MV addition caused decrease in area per molecule in case of DOPC, whereas only slight change in Liver PI, which remained almost constant with increasing pressure (Fig. 6A). Coexistence of $L_{c}+L_{e}$ phase seemed to be very mild in the isotherm for DOPC with no clear occurrence in the other two lipids. On the contrary, an increase in the molecular area of DOPG in the presence of MV was observed as evident in the rise in surface pressure (Fig. $6 \mathrm{~A}$ ). A reduction of $1.52 \mathrm{mN} / \mathrm{m}$ and $3.01 \mathrm{mN} / \mathrm{m}$ in the collapse pressure of DOPC and Liver PI membrane was observed, though DOPG membrane showed a little rise of $0.72 \mathrm{mN} / \mathrm{m}$ (Fig. $6 \mathrm{H}$ ). The negative values of the change in bending force $\left(\Delta \mathrm{F}_{\mathrm{b}}\right)$ suggest bending of monolayer (25) by the interacting MV as observed in case of both DOPC and Liver PI lipid membranes (Fig. 6F and Table S2). Further, MV interaction/fusion 
induced a reduction in monolayer compressibility modulus $\left(\mathrm{C}_{\mathrm{s}}{ }^{-1}\right)$ by around $\sim 25 \mathrm{mN} / \mathrm{m}, \sim 2 \mathrm{mN} / \mathrm{m}$ and $\sim 15 \mathrm{mN} / \mathrm{m}$, in DOPC, DOPG and Liver PI lipid membranes, respectively. (Fig. 6D). The reduction in $\mathrm{Cs}^{-1}$ in the presence of $\mathrm{MV}$ suggests a rise in the membrane monolayer compressibility. We next wanted to see if the mixing of MV with each lipid monolayer is thermodynamically favourable or not. A decreasing negative value trend in $\Delta G_{\text {Excess }}$ was observed (Fig. $6 E$ and Table S3) with respect to increasing surface pressure till $15 \mathrm{mN} / \mathrm{m}$ in DOPC and Liver PI and $10 \mathrm{mN} / \mathrm{m}$ in DOPG after which the curve rose. Upon the mixing of MV, DOPG membrane monolayer showed the lowest free energy change of $\sim-1.05 \mathrm{~kJ} / \mathrm{mol}$ and highest being $\sim-1.7 \mathrm{~kJ} / \mathrm{mol}$ for Liver PI indicating more favourable mixing with Liver PI monolayer.

We then sought to question the role of lipid acyl chain length in MV interaction/fusion using monolayer of lipids at the air-water interface. This is important as under cellular conditions transient phase separated regions differ in the membrane thickness (26). To investigate the role of chain length we have chosen zwitterionic headgroup with phosphatidylcholine having varying chain length which were DLPC, DMPC, DPPC and DSPC lipids (i.e., C12:0, C14:0, C16:0 and C18:0, respectively). The $\pi-A$ isotherms of the monolayers having lipids of various chain length formed at $25^{\circ} \mathrm{C}$ treated with bacterial MVs is shown in Fig. 6B. Analysis of surface pressure-area curves of monolayers of increasing acyl chain length, which corresponds to membrane bilayer thickness, were in good agreement with previous reports (30). We observed that the mean molecular area occupied by phospholipids decrease with increasing chain length in control conditions except DPPC i.e. in absence of bacterial MVs (Fig. 6B), which can be explained by the effective acyl chain hydrophobic interactions(31). In presence of bacterial MVs, increase in area per molecule was observed in each of the four membrane monolayers with rise in surface pressure (Fig 6B). The observed increase in molecular area was fairly constant throughout all the phases in DLPC, DPPC and DSPC membrane monolayers (Fig. 6B) whereas an increasing trend was observed in DMPC till $15 \mathrm{mN} / \mathrm{m}$ (Fig. 6B). Decrease in collapse pressure by $3.15 \mathrm{mN} / \mathrm{m}$ and $5.04 \mathrm{mN} / \mathrm{m}$ was calculated for DLPC and DMPC. Highest decrease in collapse pressure of $\sim-7 \mathrm{mN} / \mathrm{m}$ amongst all lipids was observed for DPPC after MV addition suggests most significant bending of membrane in 16:0 acyl chain length. This decline was least in case of DSPC (Fig. 6F). Fig. 6E show monolayer compressibility curves of the lipids in presence of $\mathrm{MV}$ with respect to control. A fall in compressibility modulus by around $10 \mathrm{mN} / \mathrm{m}$ was seen in DLPC, whereas the same decline was $35 \mathrm{mN} / \mathrm{m}$ in DPPC. Compressibility modulus showed a dramatic increase in case of DMPC and DSPC with a value of almost $50 \mathrm{mN} / \mathrm{m}$ and $60 \mathrm{mN} / \mathrm{m}$. The $\Delta G_{\text {Excess }}$ change though followed falling trend up to a particular pressure, there was not much difference between the energy curves of individual lipids (Fig. 6E). At $15 \mathrm{mN} / \mathrm{m}$, the free energy change was highest in DLPC followed by DPPC suggesting ideal mixing of bacterial MVs with these membranes. The lowest energy change of mixing was observed in case of DMPC throughout all pressures (Fig. 6G and Table S3).

We then investigated the role of cholesterol in bacterial MV interaction/fusion and the subsequent mechano-elastic changes in lipid monolayer. To this end, as described earlier we used different concentrations of cholesterol to make ternary lipid mix containing DOPC:BSM:Chol in 5:5:0, 4:4:2 and 3:3:4 ratio, mimicking membranes with fully disordered (5:5:0) and co-existing phase separated regions (4:4:2 and 3:3:4). $\pi-A$ isotherms of show similar trend with increase in molecular area in the presence of MV (Fig. 6C). A noticeable aspect observed in the monolayer with highest cholesterol content (3:3:4) in the presence of MV, was that - after surface pressure of around 7.5 $\mathrm{mN} / \mathrm{m}$, there was a left shift followed by a right shift after $35 \mathrm{mN} / \mathrm{m}$. Collapse pressure reduced in $0 \%$ and $40 \%$ cholesterol concentration while increased by $0.44 \mathrm{mN} / \mathrm{m}$ in the case of $20 \%$ (Fig. $6 \mathrm{H}$ and Table S2). Compressibility modulus (Fig. 6F) clearly show how increasing concentration of cholesterol induces decrease in monolayer elasticity after MV adhesion, the highest being in case 
of $40 \%$ cholesterol. $\Delta \mathrm{G}_{\text {Excess }}$ was significantly high in case of $4: 4: 2$ as compared to the other two mixtures at all pressures (Fig. 6G and Table S3).

\section{Discussion}

Despite the different physicochemical architecture of the bacterial MVs and the eukaryotic host cell membranes, membrane fusion is accepted as one of the mechanisms of MV entry $(3,11)$. In this study we reconstitute the MV interaction with GUVs and monolayer membranes mimicking various host cellular membrane conditions to quantitatively elucidate the MV mediated modulation of host membrane properties such as fluidity, dipole potential and elasticity. To do this, a non-pathogenic E. coli strain was chosen as a model Gram-negative bacteria to isolate bacterial MVs. The bacterial MVs labelled with Nile Red was found to undergo significant internalization by macrophage and fusion with liposomes (Fig. 1I \& Fig. 2A), in line with previous observations (10, 13). Fusion of bacterial MVs to host membranes is involved in transmission of virulence factors in non-phagocytic cells (15) as well as in reprogramming of phagosomes in phagocytic macrophage $(32,33)$. The homogenous binding of DiO-labelled bacterial MVs to the reconstituted lipid membranes mimicking the outer-leaflet of the host cell membrane (Fig. 2A), and the observed monolayer area expansion accompanied by an ideal mixing reflected in a negative free energy change suggest the fusion of MV although adhesion not progressing into complete fusion cannot be totally ruled out (Fig. 2D, $2 \mathrm{~F}$ ). We then examined the changes in the host membrane fluidity and dipole potential by incorporating a potentiometric dye in membranes, upon the interaction of MVs. We show quantitative evidence that the fusion of bacterial MVs results in enhanced fluidization (depicted in the decrease in fluorescence anisotropy of the dye) and increased dipole potential of the host model membrane (Fig. 2B-2C). The observed range of dipole potential of reconstituted lipid membranes were in line with previous reports (34). The membrane surface potential of bacterial MVs and lipid membranes was found to be negative (Table S1). Such trans-negative membrane surface potential was reported to be essential for cell-cell fusion $(35,36)$, also supported by the observed increase in the dipole potential of host model membrane (OLMM) upon the addition of more dipoles from bacterial MVs (Fig. 2C). The observed fluidization is supported by the experimentally determined increase in molecular area per lipid as well as elasticity (as seen in reduction in the $\mathrm{Cs}_{\mathrm{s}}^{-1}$ at surface pressure at $35 \mathrm{mN} / \mathrm{m}$ ) (Fig. 2D-2E). The surface-pressure area isotherm of the lipid fraction of MV devoid of any proteins suggests that the MV membrane is extremely rigid as evident from the low collapse pressure (Fig. S2). LPS, the major component of MV owing to its relatively wider crosssection area and low head-to-tail aspect ratio could be a major contributor towards fluidization as it is known to uniformly adhere and incorporate in egg-PC, DOPG and DOPE containing lipid bilayer (37) all of which are present in the OLMM. This is in line with recent finding that Phthiocerol dimycocerosates (DIMs), a lipid component of Mycobacterium tuberculosis, are transferred to and accommodated in the host cell membrane due to its conical shape (38).

The overall change in the fluidity of the host cell membrane would depend on the degree of interaction between bacterial MVs and the lipids specificity in the OLMM. This could further be influenced by both the lipid head group and the acyl chain length. Indeed, the fusion of bacterial MVs resulted in contrasting effects on fluidity and dipole potential of different lipid membranes. MV fusion to DOPC, Liver PI and DOPG but not BSM membrane, results in enhanced membrane fluidity as well as increased dipole potential arising due to adhesion and incorporation of bacterial MVs (Fig. 3, and Fig. S3). Slight decrease in area per lipid molecule in case of DOPC and Liver PI membranes suggests adhesion and mixing of lipids from bacterial MVs into monolayer membranes, unlike in case of DOPG membranes, where area expansion was observed suggesting significant interaction with headgroups (Fig. 6A), reflected in the respective change in elasticity and bending force (Fig. 6D and $6 \mathrm{H}$ ). The changes in area per lipid molecule are coupled to the degree of interfacial adhesion/fusion of the interacting components $(19,39)$. The observed increase in area per lipid molecule is likely due to the formation of inverted cubic structure/aggregates at the adhesion sites resulting in expulsion of some lipids $(37,38,40)$, which, also explains the observed 
relatively higher negative values of $\triangle G_{\text {Excess. }}$ Likewise, $M V$ fusion to only DPPC membrane results in enhanced fluidization accompanied by increase in interfacial molecular dipoles (Fig. 4C). On the contrary, unlike DPPC (a C16 lipid) both shorter and longer acyl chain lengths in DLPC, DMPC and DSPC membrane seem to undergo significant lowering of fluidity as well as largely unchanged to slight increase in dipole potential (Fig. 4). Incubation of monolayers with bacterial MVs showed that bacterial MVs have ideal mixing with these lipids at the interfaces as Gibbs free energy mixing was negative in all these membranes (Fig. 6G and Table S3). Maximum change in the bending force and in plane elasticity was observed for DPPC monolayer compared to other lipid monolayers (Fig. $6 \mathrm{~B}$, Fig. $6 \mathrm{H}$ and Table S2). Together, the data suggest that while MV interaction is facilitated by both zwitterionic and negatively charged head groups, however, an optimum acyl chain length is essential for effective interaction/fusion in line with previous observations (41).

Lipid rafts have also been reported to facilitate the fusion of bacterial MVs (9). However, this was proved using cholesterol depleting or sequestering agents such as filipin or Methyl-ß-cyclodextrin, which cannot rule out the possibility that disruption of cholesterol on a large scale may affect protein dependent processes not limited to lipid rafts. One way to examine and correlate the exclusive dependence of MV mixing with lipid rafts is by reconstituting minimal systems of lipid raft-like regions without any protein. Bacterial $\mathrm{MVs}$ are found to show strongest adhesion to fully disordered membranes devoid of cholesterol (DOPC:BSM:Chol, 5:5:0) accompanied by highest decrease in the fluidity (Fig. 5A and 5B). Further, increase in the cholesterol in the membrane lead to decreased adhesion of bacterial MVs to the membrane (Fig. 5D and 5G). This is supported by the observed molecular area per lipid expansion observed in surface pressure - area isotherms. The slight expansion in molecular area per lipid seen in fully disordered membrane (DOPC:BSM:Chol, 5:5:0) suggest expulsion of lipids due to steric pressure within monolayer during the adhesion of bacterial MVs at multiple sites (Fig. 6C). Likewise, largest expansion in molecular area per lipid was observed in case of (DOPC:BSM:Chol, 4:4:2) hinting at a strong expulsion of monolayer lipids during the adhesion of bacterial MVs, accompanied by a reduction in the dipole potential of the membrane (Fig. $5 \mathrm{D}$, and $5 \mathrm{~F}$ and $6 \mathrm{C}$ ). This is further supported by positive $\Delta \mathrm{G}$ Excess and a positive $\Delta \mathrm{F}_{\mathrm{b}}$ that suggest unfavourable non-ideal mixing (Fig. 6G, and $6 \mathrm{H}$ ). MV adhesion to membranes containing higher concentration of cholesterol (DOPC:BSM:Chol, 3:3:4) is relatively weaker compared to fully disordered membranes (Fig. 5G). The increasing cholesterol levels results in coexisting phase separating regions ( $L_{o}$ and $L_{d}$ ) within the (DOPC:BSM:Chol, 4:4:2 \& 3:3:4) membranes, however, the number of the phase separating boundaries is higher in the former. Such differences in the phase separating boundaries would accumulate different degrees of interfacial energy in the membrane, that would facilitate the adhesion of bacterial MVs, which explains the observed variation (42). Cholesterol amounting to low to moderate levels will not only increase the order in a fluid membrane but also trigger formation of phase separated regions with higher line tension that facilitates the adhesion and fusion of bacterial MVs $(42,43)$. On the contrary, higher cholesterol level results in formation of phase separated regions that are larger in size and less in number, reducing the overall interfacial energy and line tension at the phase boundaries that hinder the bacterial MVs fusion to host membrane (Fig. 5G). Our results suggest the lipid head-group preference, elasticity and line tension in membranes showing phase separation could drive the fusion of bacterial MVs to the host membranes.

Increase in the dipole potential of host lipid membrane by fusion of MV is likely to stimulate conformational changes in the membrane bound/inserted receptor proteins $(28,44)$. Such conformational changes in transmembrane receptor proteins have been reported to significantly alter the receptor signalling in host cells (45). Additionally, our study also suggests that MV interaction with lipid rafts/phase boundaries may have an important role in altering host cell signalling (46). Very recently, Mycobacterium ulcerans endotoxin, Mycolactone, was shown to have potent effect on reorganization of raft-like model membranes (47). Likewise, Chlamydia pneumoniae effector protein $\mathrm{CPn} 0678$ was shown to induce curvature in the host membrane during its fusion (48). Further, bacterial membrane vesicles were found to activate anti-tumour response by inducing production of IFN- $\gamma$ (49). It would be interesting to investigate the role of MV induced changes in the dipole potential and elasticity of phagosomal membranes, on the phagosome maturation that involve acidification and fusion with lysosomal vesicles (50). Although our 
observations are based on a non-pathogenic strain of $E$. coli as a model bacteria, it is important to note that similar observations can be expected with pathogenic $E$. coli as they share more than $70 \%$ similarity in the protein content of the their membrane vesicles $(51,52)$. Further, it is noteworthy that this study focuses on a holistic picture of the consequence of whole MV interaction on host membrane without dissecting the role individual components of MV that may or may not contribute to the observed modulation of host membrane properties. Identifying the potential proteins in the MVs and their contribution to the modulation of the host cell membrane remains an important aspect that would require extensive research. 


\title{
Materials and Methods
}

\begin{abstract}
Membrane Vesicles Purification from E. coli
For membrane vesicle isolation, Gram negative bacteria, E. coli were used. The methodology for $M V$ vesiculation and isolation was adopted from the procedure described by Sezgin et. al. (53) with some modification and optimization that allowed us to obtain reasonably homogenous pool of MV size in the range of 50-150 nm. We compared the MVs isolated by chemical vesiculation method with the native method of ultracentrifugation which is already established. Briefly in this method bacteria were harvested to OD 1.0 then cells were palleted twice in MV buffer $(\mathrm{pH}-7.4)$ at 5000 $\mathrm{rpm}$ for 10 minutes at $4{ }^{\circ} \mathrm{C}$ temperature. Bacteria were resuspended in with $3 \mathrm{mM}$ of $\mathrm{N}$ ethylmaleimide (NEM) in MV buffer and kept at room temperature for 1 hour for the vesiculation of membrane vesicles (MVs) form bacterial cell membrane. NEM treated bacteria now centrifuged at $18000 \mathrm{~g}$ for 2 hours at $4{ }^{\circ} \mathrm{C}$ to pallet the bacterial cells and debris in the MV buffer and the supernatant containing MVs was collected. MVs in supernatant were concentrated using $10 \mathrm{kD}$ cut of concentrator (Millipore) and filtered through $0.22 \mu \mathrm{M}$ filter paper for the size homogeneity. The filtered solution containing MVs were collected and stored in $-20{ }^{\circ} \mathrm{C}$ for its characterization and further experimental analysis. MVs isolation through ultracentrifugation is discussed in detail in supplementary information.
\end{abstract}

\section{Fluorescence Labeling of Bacterial MVs}

Purified bacterial MVs were labelled with a lipophilic dye DiO to study binding of bacterial MVs with reconstituted membranes. DiO dye mixed to MV in ratio of 1:100 v/v dropwise with constant stirring at $37^{\circ} \mathrm{C}$ for 30 minutes for incorporation of dye in bacterial MVs. To separate unlabeled dye from labelled bacterial MVs, the mixture was washed with PBS buffer four times using 10kD cut-off concentrators (Merck-millipore). Labelled bacterial MVs were kept at $4^{\circ} \mathrm{C}$ and can be stored upto one week for further experiments.

\section{Reconstitution of Fluorescence Labelled Giant Unilamellar Vesicles (GUVs)}

Giant unilamellar vesicles (GUVs) were prepared using polyvinyl alcohol (PVA) gel-assisted method as previously described by Weinberger et. al. with slight modifications (54). PVA-coating on the surface of glass slides were used as film for swelling of lipid film for reconstitution of GUVs. To prepare PVA coated slides, PVA was dissolved in deionized water in $5 \% \mathrm{w} / \mathrm{w}$ ratio of PVA/water with constant stirring at $90^{\circ} \mathrm{C}$. From this PVA solution, $200-300 \mu \mathrm{L}$ is evenly spread on the glass slide and dried in oven at $50^{\circ} \mathrm{C}$ for 30 minutes which makes a thin film over the surface of slide. This dried PVA-coated slides then cleaned with UV for 15 minutes to prevent dewetting of PVA coating. Now, $20 \mu \mathrm{L}$ of Lipid solution in chloroform $(1 \mathrm{mg} / \mathrm{mL})$ doped with 1 mole \% Rhodamine-PE of total lipid mole fraction was smeared on PVA-coated slide with Hamilton syringe. Residual chloroform on the PVA-coated slide was evaporated by keeping the slide in vacuum chamber for 2 to 3 hours. A chamber on the lipid spread PVA-coated slide was made using nitrile ring sealed by using a second coverslip and clips as sealant. This lipid film was then swelled by filling the chamber with $1 \mathrm{~mL}$ of $10 \mathrm{mM}$ phosphate buffer saline (PBS) at pH of 7.4 for vesiculation. After 30 minutes the chamber was gently agitated and the buffer of chamber was collected in microcentrifuge tubes containing GUVs.

\section{Statistical Analysis}

Data were analyzed by one-way analysis of variance (ANOVA) using Origin 8.5 software. $p$-values $<0.5$ were considered were considered statistically significant. All values were reported as mean \pm SE from appropriate sample size and three independent experiments as indicated and where $\left({ }^{* * *}\right)$ depicts $p<0.001,\left({ }^{* *}\right) p<0.01$ and $\left(^{*}\right) p<0.05$.

Methodology for characterization of MVs by, Internalization in macrophage cells, confocal imaging for affinity of MVs with host lipid membranes, reconstitution of Di-8-ANEPPS doped large unilamellar vesicles (LUVs), measurements of dipole potential and anisotropy, and langmuir 
Blodgett monolayer preparation and isotherm analysis are available in Material and Method section of SI appendix.

\section{Acknowledgements}

We would like to thank Dr Hirak Chakraborty, Dr Yusuf Akhter and Dr Bhavani Shankar Sahu for critical reading and stimulating discussion on the manuscript. We would like to gratefully acknowledge the financial support received from the Department of Science and Technology (EMR/2017/004513) and Department of Biotechnology, Govt. of India (BT/PR/21226/MED/122/41/2016). AP, AT and TM acknowledge MHRD for the GATE fellowship.

\section{Declaration of Interests}

Authors declare no competing interests. 


\section{References}

1. Beveridge TJ (1999) Structures of gram-negative cell walls and their derived membrane vesicles. Journal of bacteriology 181(16):4725-4733.

2. Schwechheimer C \& Kuehn MJ (2015) Outer-membrane vesicles from Gram-negative bacteria: biogenesis and functions. Nature Reviews Microbiology 13(10):605.

3. Kulp A \& Kuehn MJ (2010) Biological functions and biogenesis of secreted bacterial outer membrane vesicles. Annual review of microbiology 64:163-184.

4. Toyofuku M, Nomura N, \& Eberl L (2018) Types and origins of bacterial membrane vesicles. Nature Reviews Microbiology:1.

5. Koeppen K, Hampton TH, Jarek M, Scharfe M, Gerber SA, et al. (2016) A novel mechanism of host-pathogen interaction through sRNA in bacterial outer membrane vesicles. PLoS pathogens 12(6):e1005672.

6. MacDonald IA \& Kuehn MJ (2012) Offense and defense: microbial membrane vesicles play both ways. Research in microbiology 163(9-10):607-618.

7. Chattopadhyay MK \& Jagannadham MV (2015) Vesicles-mediated resistance to antibiotics in bacteria. Frontiers in microbiology 6:758.

8. Kaparakis-Liaskos M \& Ferrero RL (2015) Immune modulation by bacterial outer membrane vesicles. Nature reviews Immunology 15(6):375.

9. Bomberger JM, MacEachran DP, Coutermarsh BA, Ye S, O'Toole GA, et al. (2009) Longdistance delivery of bacterial virulence factors by Pseudomonas aeruginosa outer membrane vesicles. PLoS pathogens 5(4):e1000382.

10. Jäger J, Keese S, Roessle M, Steinert M, \& Schromm AB (2015) Fusion of L egionella pneumophila outer membrane vesicles with eukaryotic membrane systems is a mechanism to deliver pathogen factors to host cell membranes. Cellular microbiology 17(5):607-620.

11. O'donoghue EJ \& Krachler AM (2016) Mechanisms of outer membrane vesicle entry into host cells. Cellular microbiology 18(11):1508-1517.

12. Greenspan P, Mayer EP, \& Fowler SD (1985) Nile red: a selective fluorescent stain for intracellular lipid droplets. The Journal of cell biology 100(3):965-973.

13. Wang X, Eagen WJ, \& Lee JC (2020) Orchestration of human macrophage NLRP3 inflammasome activation by Staphylococcus aureus extracellular vesicles. Proceedings of the National Academy of Sciences.

14. Van Meer G, Voelker DR, \& Feigenson GW (2008) Membrane lipids: where they are and how they behave. Nature reviews Molecular cell biology 9(2):112.

15. O'Donoghue EJ, Sirisaengtaksin N, Browning DF, Bielska E, Hadis M, et al. (2017) Lipopolysaccharide structure impacts the entry kinetics of bacterial outer membrane vesicles into host cells. PLoS pathogens 13(11):e1006760.

16. Bandari S, Chakraborty H, Covey DF, \& Chattopadhyay A (2014) Membrane dipole potential is sensitive to cholesterol stereospecificity: implications for receptor function. Chemistry and physics of lipids 184:25-29.

17. Gross E, Bedlack Jr RS, \& Loew LM (1994) Dual-wavelength ratiometric fluorescence measurement of the membrane dipole potential. Biophysical journal 67(1):208-216.

18. Starke-Peterkovic T, Turner N, Vitha MF, Waller MP, Hibbs DE, et al. (2006) Cholesterol effect on the dipole potential of lipid membranes. Biophysical journal 90(11):4060-4070.

19. Brockman H (1999) Lipid monolayers: why use half a membrane to characterize proteinmembrane interactions? Current opinion in structural biology 9(4):438-443. 
20. Allende D, Vidal A, \& Mclntosh TJ (2004) Jumping to rafts: gatekeeper role of bilayer elasticity. Trends in biochemical sciences 29(6):325-330.

21. Brown RE \& Brockman HL (2007) Using monomolecular films to characterize lipid lateral interactions. Lipid Rafts, (Springer), pp 41-58.

22. Smaby JM, Kulkarni VS, Momsen M, \& Brown RE (1996) The interfacial elastic packing interactions of galactosylceramides, sphingomyelins, and phosphatidylcholines. Biophysical journal 70(2):868-877.

23. Feng S-s (1999) Interpretation of mechanochemical properties of lipid bilayer vesicles from the equation of state or pressure- area measurement of the monolayer at the airwater or oil- water interface. Langmuir 15(4):998-1010.

24. Goodrich F (1957) Proceedings, 2nd International Congress on Surface Activity. Vol. I:85.

25. Peetla C, Jin S, Weimer J, Elegbede A, \& Labhasetwar V (2014) Biomechanics and thermodynamics of nanoparticle interactions with plasma and endosomal membrane lipids in cellular uptake and endosomal escape. Langmuir 30(25):7522-7532.

26. Lewis BA \& Engelman DM (1983) Lipid bilayer thickness varies linearly with acyl chain length in fluid phosphatidylcholine vesicles. Journal of molecular biology 166(2):211-217.

27. Simons K \& Ikonen E (1997) Functional rafts in cell membranes. nature 387(6633):569.

28. Ermakov YA, Averbakh AZ, Yusipovich Al, \& Sukharev S (2001) Dipole potentials indicate restructuring of the membrane interface induced by gadolinium and beryllium ions. Biophysical journal 80(4):1851-1862.

29. Chen D \& Santore MM (2014) Large effect of membrane tension on the fluid-solid phase transitions of two-component phosphatidylcholine vesicles. Proceedings of the National Academy of Sciences 111(1):179-184.

30. Miyoshi T \& Kato S (2015) Detailed analysis of the surface area and elasticity in the saturated 1, 2-diacylphosphatidylcholine/cholesterol binary monolayer system. Langmuir 31(33):9086-9096.

31. Zhao L \& Feng S-S (2004) Effects of lipid chain length on molecular interactions between paclitaxel and phospholipid within model biomembranes. Journal of colloid and interface science 274(1):55-68.

32. Ge J \& Shao F (2011) Manipulation of host vesicular trafficking and innate immune defence by Legionella Dot/Icm effectors. Cellular microbiology 13(12):1870-1880.

33. Hubber A \& Roy CR (2010) Modulation of host cell function by Legionella pneumophila type IV effectors. Annual review of cell and developmental biology 26:261-283.

34. Wang L, Bose PS, \& Sigworth FJ (2006) Using cryo-EM to measure the dipole potential of a lipid membrane. Proceedings of the National Academy of Sciences 103(49):1852818533.

35. Asawakarn T, Cladera J, \& O'Shea P (2001) Effects of the membrane dipole potential on the interaction of saquinavir with phospholipid membranes and plasma membrane receptors of Caco-2 cells. Journal of Biological Chemistry 276(42):38457-38463.

36. Samsonov AV, Chatterjee PK, Razinkov VI, Eng CH, Kielian M, et al. (2002) Effects of membrane potential and sphingolipid structures on fusion of Semliki Forest virus. Journal of virology 76(24):12691-12702.

37. Nomura K, Inaba T, Morigaki K, Brandenburg K, Seydel U, et al. (2008) Interaction of lipopolysaccharide and phospholipid in mixed membranes: solid-state 31P-NMR spectroscopic and microscopic investigations. Biophysical journal 95(3):1226-1238. 
38. Augenstreich J, Haanappel E, Ferré G, Czaplicki G, Jolibois F, et al. (2019) The conical shape of DIM lipids promotes Mycobacterium tuberculosis infection of macrophages. Proceedings of the National Academy of Sciences 116(51):25649-25658.

39. Mura M, Dennison SR, Zvelindovsky AV, \& Phoenix DA (2013) Aurein 2.3 functionality is supported by oblique orientated $\alpha$-helical formation. Biochimica et Biophysica Acta (BBA)Biomembranes 1828(2):586-594.

40. Adams PG, Lamoureux L, Swingle KL, Mukundan H, \& Montaño GA (2014) Lipopolysaccharide-induced dynamic lipid membrane reorganization: tubules, perforations, and stacks. Biophysical journal 106(11):2395-2407.

41. Szule JA, Fuller NL, \& Rand RP (2002) The effects of acyl chain length and saturation of diacylglycerols and phosphatidylcholines on membrane monolayer curvature. Biophysical journal 83(2):977-984.

42. Yang S-T, Kiessling V, \& Tamm LK (2016) Line tension at lipid phase boundaries as driving force for HIV fusion peptide-mediated fusion. Nature communications 7:11401.

43. Risselada HJ, Marelli G, Fuhrmans M, Smirnova YG, Grubmüller H, et al. (2012) Linetension controlled mechanism for influenza fusion. PLoS One 7(6):e38302.

44. Sukharev SI, Sigurdson WJ, Kung C, \& Sachs F (1999) Energetic and spatial parameters for gating of the bacterial large conductance mechanosensitive channel, MscL. The Journal of general physiology 113(4):525-540.

45. Zhou Y, Wong C-O, Cho K-j, Van Der Hoeven D, Liang H, et al. (2015) Membrane potential modulates plasma membrane phospholipid dynamics and K-Ras signaling. Science 349(6250):873-876.

46. Kovács T, Batta G, Hajdu T, Szabó Á, Váradi T, et al. (2016) The dipole potential modifies the clustering and ligand binding affinity of ErbB proteins and their signaling efficiency. Scientific reports 6:35850.

47. Nitenberg M, Benarouche A, Maniti O, Marion E, Marsollier L, et al. (2018) The potent effect of mycolactone on lipid membranes. PLoS pathogens 14(1):e1006814.

48. Hänsch S, Spona D, Murra G, Köhrer K, Subtil A, et al. (2020) Chlamydia-induced curvature of the host-cell plasma membrane is required for infection. Proceedings of the National Academy of Sciences 117(5):2634-2644.

49. Kim OY, Park HT, Dinh NTH, Choi SJ, Lee J, et al. (2017) Bacterial outer membrane vesicles suppress tumor by interferon- $\gamma$-mediated antitumor response. Nature communications 8(1):626.

50. Steinberg BE, Touret N, Vargas-Caballero M, \& Grinstein S (2007) In situ measurement of the electrical potential across the phagosomal membrane using FRET and its contribution to the proton-motive force. Proceedings of the National Academy of Sciences 104(22):9523-9528.

51. Lee EY, Bang JY, Park GW, Choi DS, Kang JS, et al. (2007) Global proteomic profiling of native outer membrane vesicles derived from Escherichia coli. Proteomics 7(17):31433153.

52. Scorza FB, Doro F, Rodríguez-Ortega MJ, Stella M, Liberatori S, et al. (2007) Proteomic characterization of outer membrane vesicles from the extraintestinal pathogenic Escherichia coli toIR IHE3034 mutant. Molecular \& Cellular Proteomics.

53. Sezgin E, Kaiser H-J, Baumgart T, Schwille P, Simons K, et al. (2012) Elucidating membrane structure and protein behavior using giant plasma membrane vesicles. nature protocols 7(6):1042. 

not certified by peer review) is the author/funder. All rights reserved. No reuse allowed without permission.

54. Weinberger A, Tsai F-C, Koenderink GH, Schmidt TF, Itri R, et al. (2013) Gel-assisted formation of giant unilamellar vesicles. Biophysical journal 105(1):154-164. 
Figure 1.

A

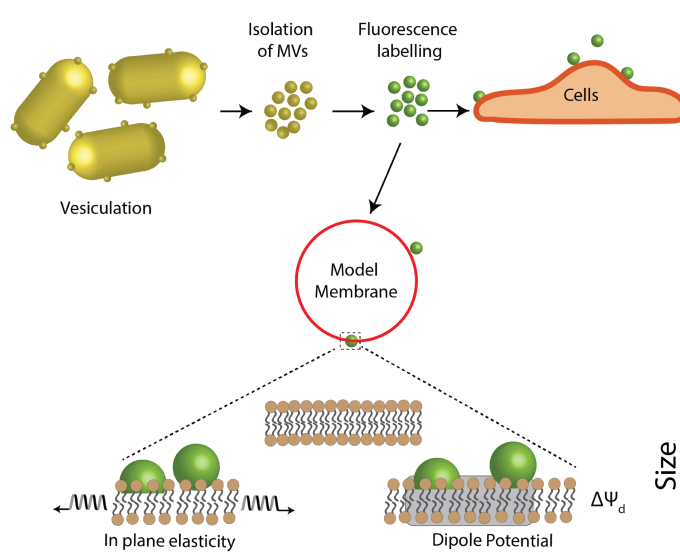

B

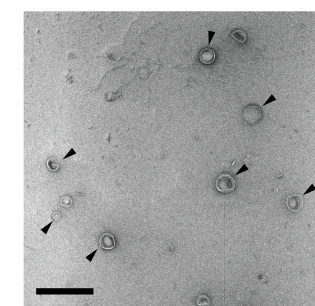

D

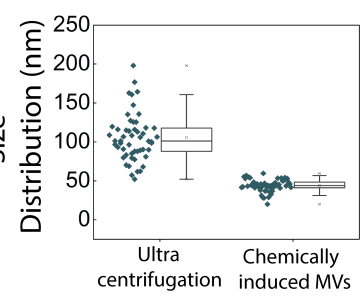

C

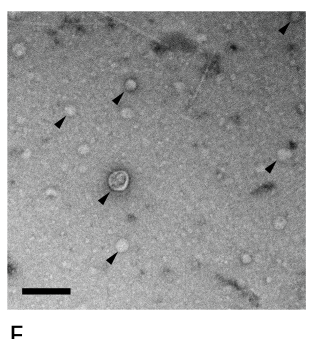

$\mathrm{E}$

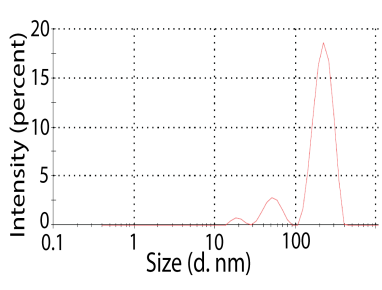

$\mathrm{F}$

G

$\mathrm{H}$
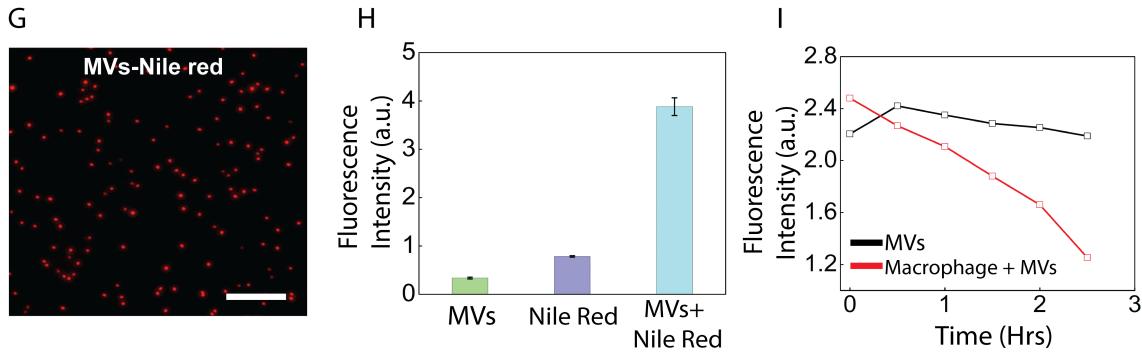

Figure 1. Characterization of bacterial Membrane Vesicles (MVs) isolated from Escherichia coli and internalization of bacterial MVs in macrophages. (A) Schematic summarizing the methodology used to isolate, fluorescently label and study the MV-host membrane interaction. (B) TEM micrograph of bacterial membrane vesicles isolated by ultracentrifugation (scale bar is 500 $\mathrm{nm}$ ). (C) TEM micrograph of MVs isolated through chemical vesiculating method (scale bar -500 $\mathrm{nm}$ ). (D) Box plot for size distribution of MVs obtained through TEM micrographs. (E) Size distribution of bacterial MVs measured by dynamic light scattering. (F) Confocal image of DiO labelled MV suspension (Scale bar - $10 \mu \mathrm{m}$ ). (G) Fluorescence image of MVs labelled with Nile red dye (scale bar is $10 \mu \mathrm{m})(\mathrm{H})$ Comparative fluorescence intensities of unlabelled Bacterial MVs, Nile red and Bacterial MVs incubated with Nile red which increased in presence of Bacterial MVs. (I) Fluorescence intensity of Nile red labelled Bacterial MVs (Black line) and Nile red labelled Bacterial MVs incubated with macrophages to confirm uptake of Bacterial MVs by the macrophage for 2.5 hours (Red line). 
Figure 2
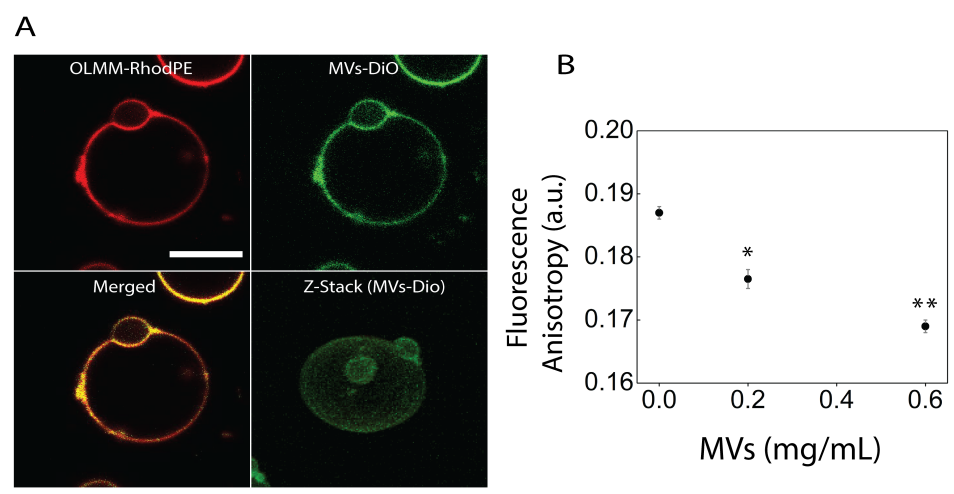

$\mathrm{D}$

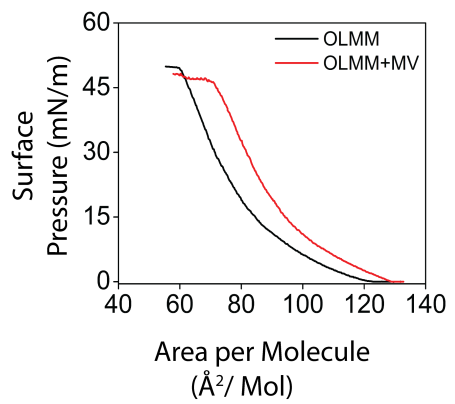

E

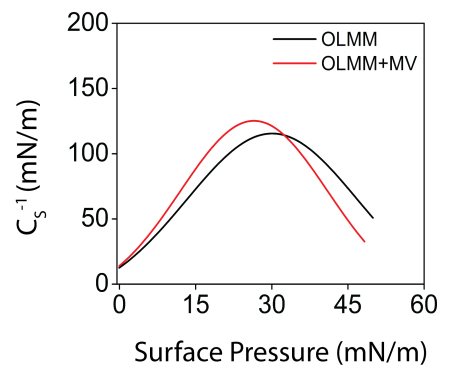

$\mathrm{C}$

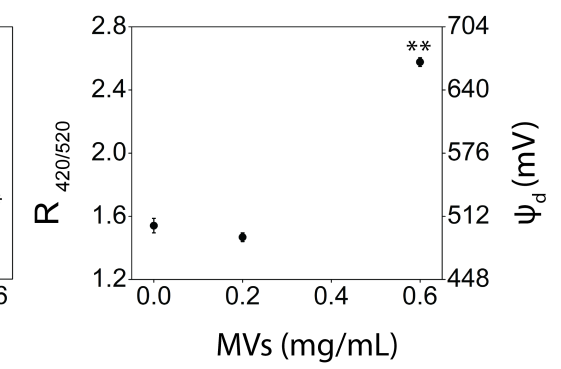

$\mathrm{F}$

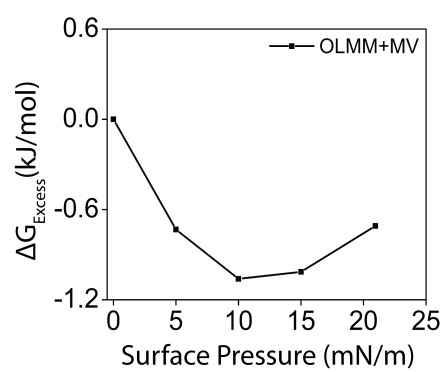

Figure 2. Effect of MVs on membrane binding, fluidity, dipole potential and Surface Pressure $(\pi)$ - Mean molecular area (A) isotherm, compressibility modulus and excess Gibb's free energy change of reconstituted outer leaflet model membrane of host cell. (A) Confocal images of reconstituted GUVs of outer leaflet model membrane (OLMM-RhodPE) composed of DOPC 35 mole $\%$, Liver PI 5 mole \%, DOPE 5.6 mole \%, Brain SM 20 mole \%, Cholesterol 30 mole \% doped with 1 $\%$ Rhodamine PE (red channel) mimicking outer leaflet of eukaryotic cell membrane. Bacterial MVs labelled with DiO binding to the GUVs (green channel) distributed homogenously over the equatorial plane of GUVs along with merged image of both red and green channels showing a co-localisation. 3D-stack of the OLMM GUVs (Green channel). Fluorescence imaging were done thrice independently

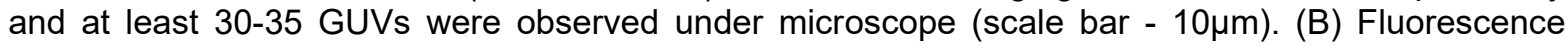
anisotropy of OLMM decreases with increasing concentration of Bacterial MVs correlating with the increased fluidity of host model membrane. (C) Fluorescence intensity ratio at $420 / 520 \mathrm{~nm}$ of wavelength and dipole potential of the outer leaflet model membrane incubated with increasing concentration of Bacterial MVs, increased at high concentration of Bacterial MVs. (D) Surface Pressure $(\pi)$ - Mean molecular area (A) isotherm of OLMM monolayer (black curve) and OLMM incubated with bacterial MVs (red curve) showing right shift. All isotherms were compressed with constant speed of 8 $\mathrm{mm} /$ minute at $25^{\circ} \mathrm{C}$ temperature. (E) Compressibility modulus (in-plane elasticity, $\mathrm{Cs}^{-1}$ ) with respect to surface pressure of OLMM monolayer (black curve) and OLMM with MV (red curve) showing decreased $\mathrm{C}_{\mathrm{s}}{ }^{-1}$ on the air-water interface. (F) Excess Gibb's free energy of mixing between OLMM and bacterial MVs shows an ideal mixing and decrease with increasing surface pressure calculated from Langmuir isotherms. All fluorescence spectroscopic data are shown as mean \pm S.E. from three independent experiments. Significant variations were compared through one-sided ANOVA of OLMM treated with $0.2 \mathrm{mg} / \mathrm{mL}$ and $0.6 \mathrm{mg} / \mathrm{mL}$ of Bacterial $\mathrm{MVs}$ with respect to control, where, $\left({ }^{* * *}\right)$ depicts $p<0.001,\left(^{* *}\right) p<0.01$ and $\left(^{*}\right) p<0.05$, data point with no asterisk mark are not significant. All representative isotherms are the mean of independently performed experiments. For isotherms and compressibility modulus curves the estimated standard error for data points of surface pressure $(\pi)$ with respect to area per molecule (A) was approximately $\pm 0.05 \mathrm{mN} / \mathrm{m}$. 
Figure 3.

A

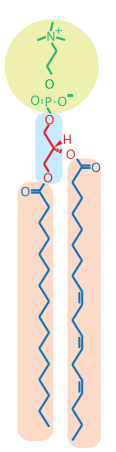

DOPC

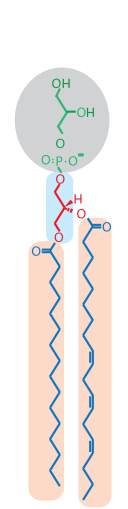

DOPG

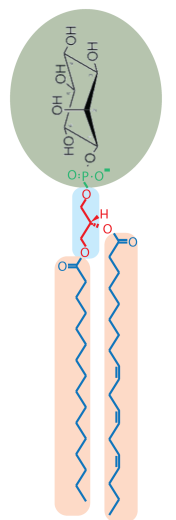

Liver PI
B Rhodamine-

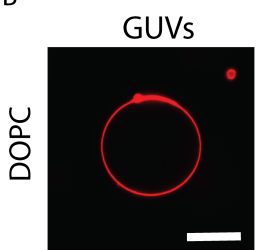

DiO-MVs
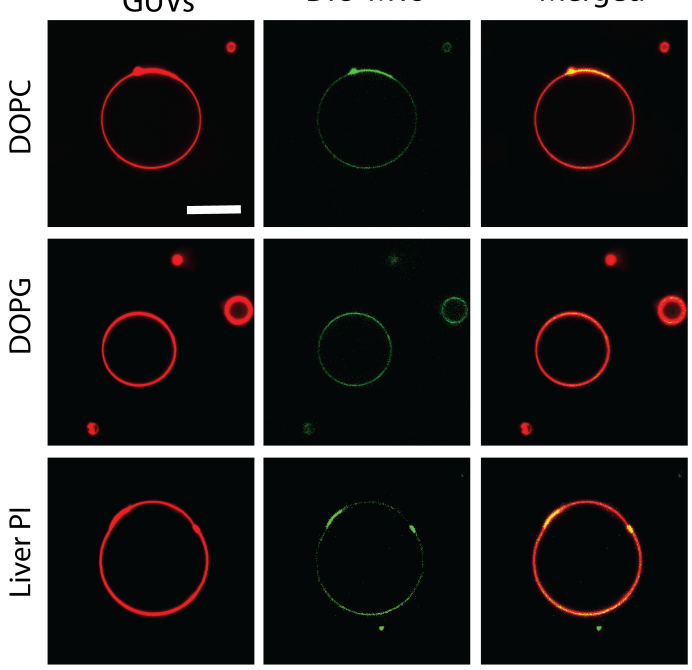

$E$

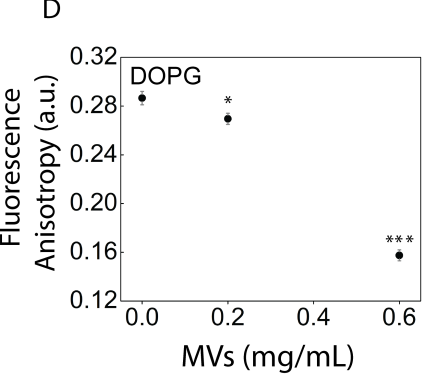

G

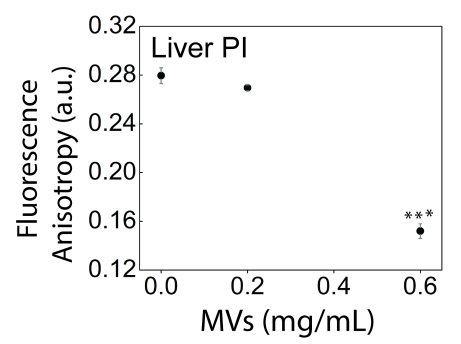

$\mathrm{H}$

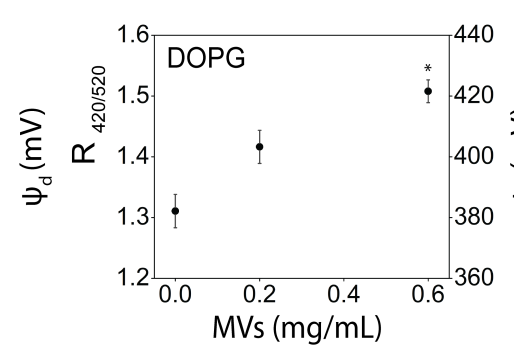

Figure 3. Monitoring the lipid specificity of host membrane for bacterial MVs interaction using confocal and the fluorometry of the potentiometric dye, Di-8-ANEPPS. (A) Biochemical structures of phospholipids- DOPC, DOPG and Liver PI. (B) Fluorescence image of DOPC, DOPG and Liver PI doped with $1 \%$ Rhodamine PE (red fluorescence) mixed with bacterial MVs labelled with DiO (green fluorescence) to visualize the interaction of bacterial MVs with lipid membrane. A green fluorescence signal on the equatorial plane of membranes suggest the interaction of bacterial MVs with these phospholipids. (C) Membrane fluidity of DOPC decreased with increasing concentration of bacterial MVs i.e. $0 \mathrm{mg} / \mathrm{mL}, 0.2 \mathrm{mg} / \mathrm{mL}$ and $0.6 \mathrm{mg} / \mathrm{mL}$. (D) Anisotropy of DOPG decreased with increasing 
concentration of bacterial MVs. (E) Liver PI membrane shows increase in fluidity with MVs treatment as compared to control. (F) Fluorescence ratio $420 / 520 \mathrm{~nm}$ and dipole potential $(\mathrm{mV})$ increased for DOPC membrane at $0.6 \mathrm{mg} / \mathrm{mL}$ concentration of bacterial MVs with respect to control DOPC membrane. (G) Dipole potential of DOPG membrane increased linearly with increasing concentration of bacterial MVs. (H) Dipole potential of Liver PI had increase at $0.6 \mathrm{mg} / \mathrm{mL}$ of MVs whereas it remained unchanged at lower concentration i.e. $0.2 \mathrm{mg} / \mathrm{mL}$. Measurements for calculation of dipole potential were done at measured intensity at excitation wavelength set at 420 and $520 \mathrm{~nm}$ respectively. All fluorescence spectroscopic data are shown as mean \pm S.E. from three independent experiments. Significant variations were compared through one-sided ANOVA of membrane condition treated with Bacterial MVs with respect to control (only lipid membrane without Bacterial MVs), where, $\left({ }^{* * *}\right)$ depicts $p<0.001,\left(^{* *}\right) p<0.01$ and $\left(^{*}\right) p<0.05$, test data point) with no asterisk mark are non-significant. 


\section{Figure 4}

A

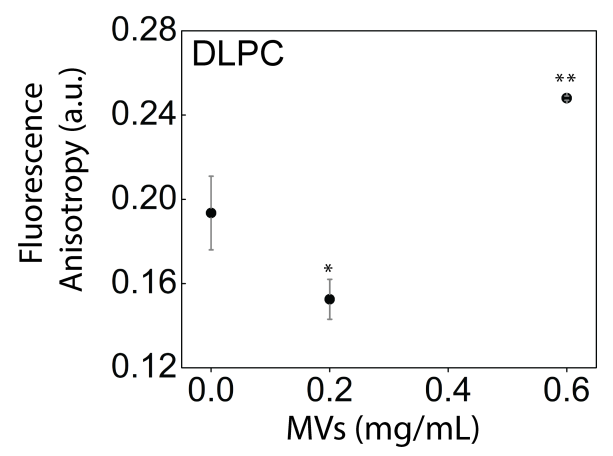

B

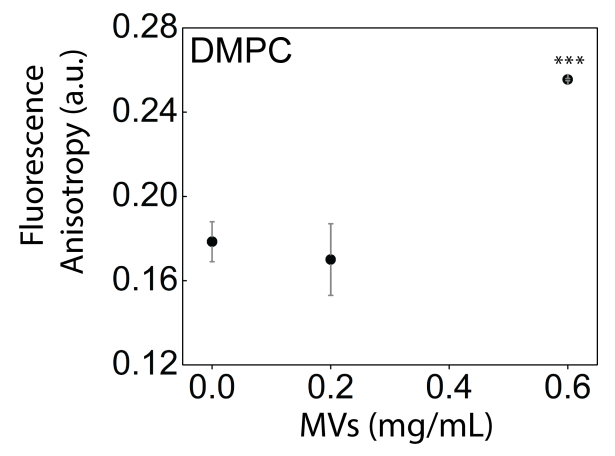

C

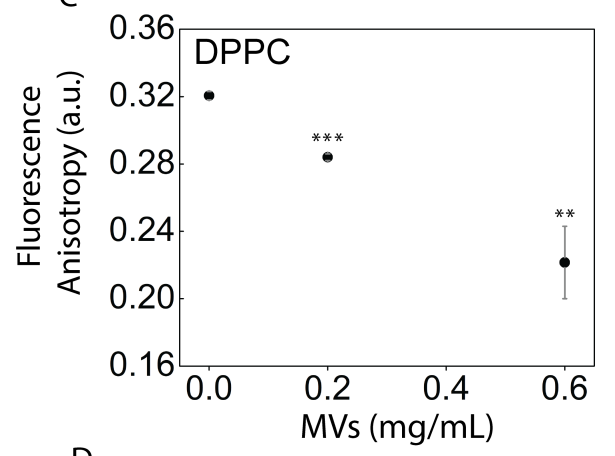

$\mathrm{D}$

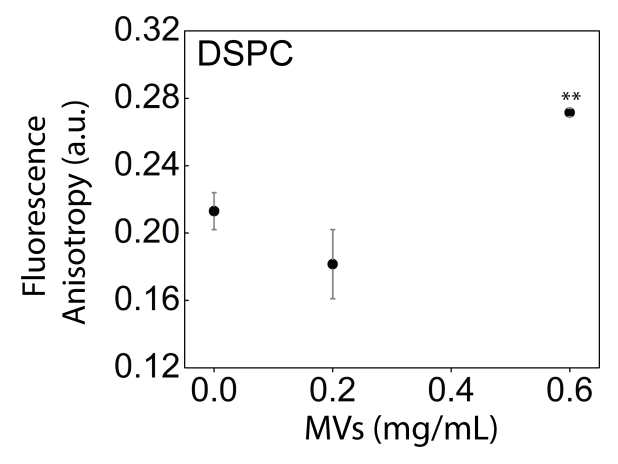

E

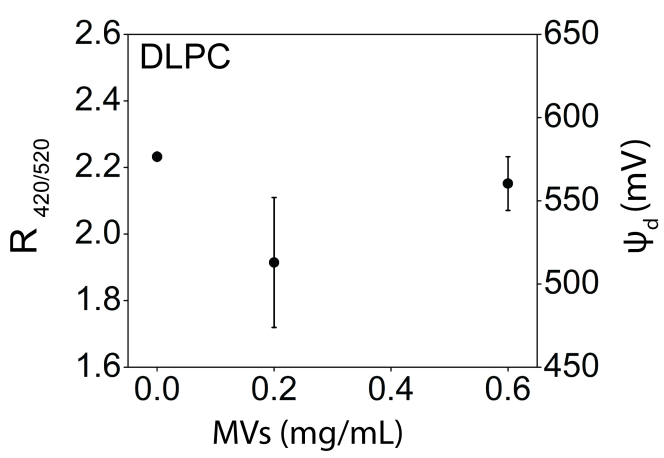

$\mathrm{F}$

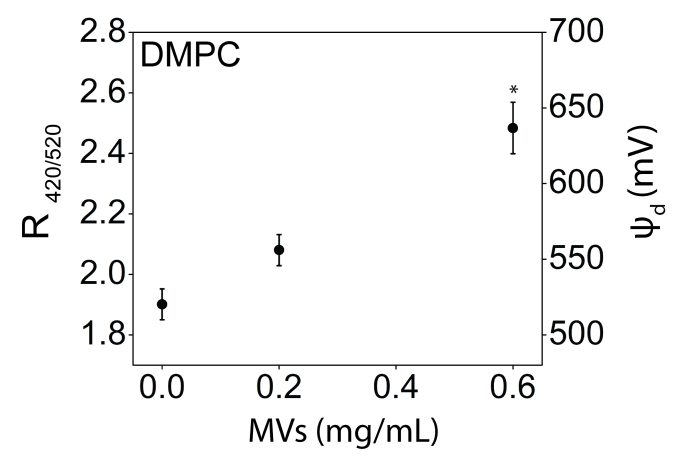

$\mathrm{G}$
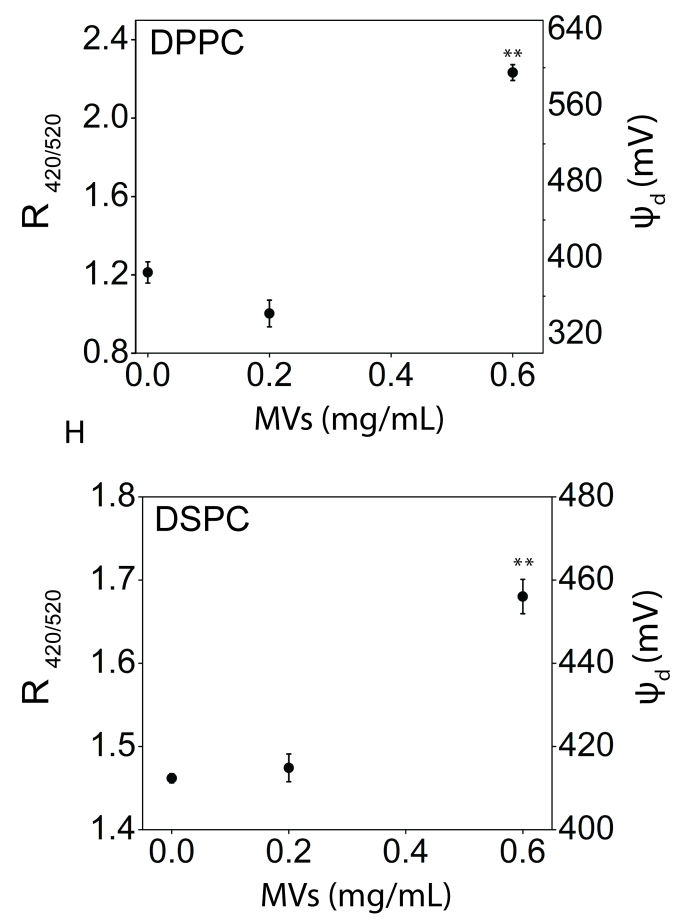
Figure 4. Variation in anisotropy and dipole potential of phospholipids with increasing bilayer thickness as a function of acyl chain length, treated with OMVs. Fluorescence anisotropy of individual lipid model membranes (A) DLPC, (B) DMPC, (C) DPPC, and (D) DSPC incubated with increasing concentration of OMVs. Changes in fluorescence ratio and dipole potential of (E) DLPC, (F) DMPC, (G) DPPC, and (H) DSPC membrane with respect to increasing concentrations of OMVs i.e. 0 $\mathrm{mg} / \mathrm{mL}, 0.2 \mathrm{mg} / \mathrm{mL}$ and $0.6 \mathrm{mg} / \mathrm{mL}$. All fluorescence spectroscopic data are shown as mean \pm S.E. from three independent experiments. Significant variations were compared through one-sided ANOVA of membrane condition treated with OMVs with respect to control (only lipid membrane without OMVs), where, $\left({ }^{* * *}\right)$ depicts $p<0.001,\left(^{* *}\right) p<0.01$ and $\left({ }^{*}\right) p<0.05$, data point with no asterisk mark are nonsignificant. 
Figure 5.

A

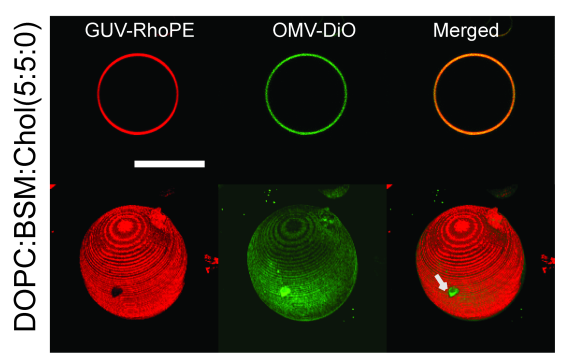

D

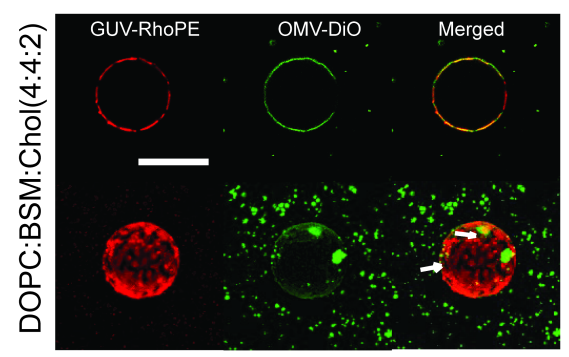

G

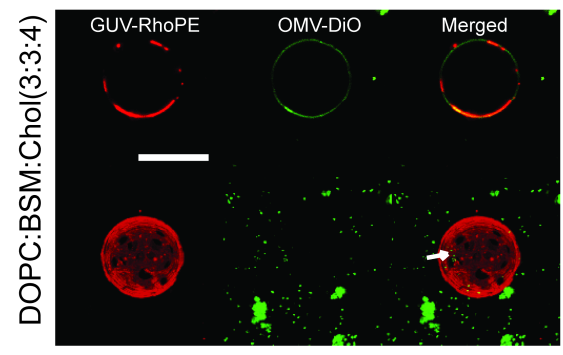

B

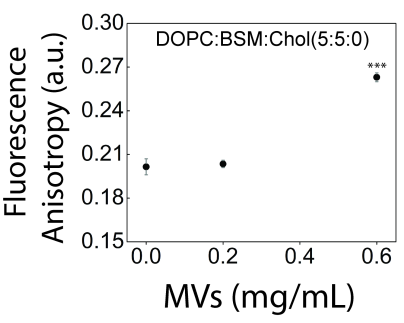

$E$

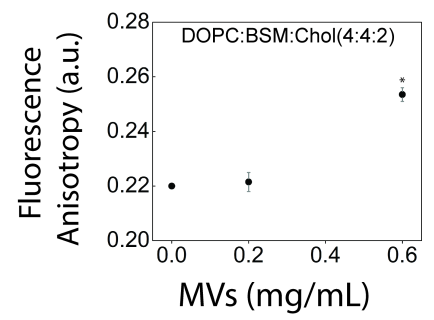

$\mathrm{H}$

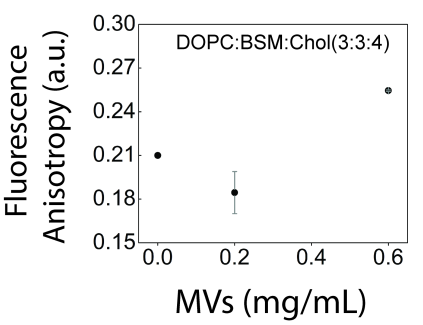

$C$

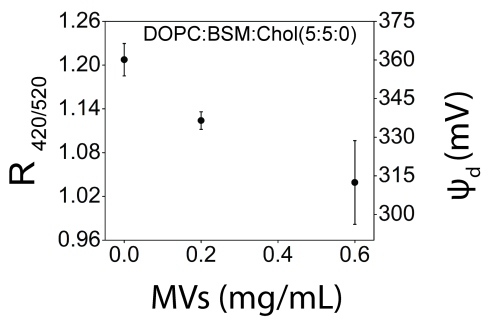

$\mathrm{F}$

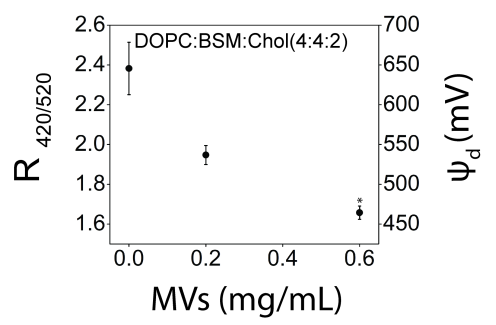

।

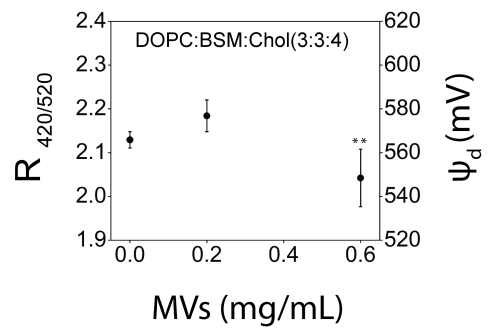

Figure 5. Effect of increasing concentration of cholesterol (liquid order) and phase behaviour of membrane on binding, changes in membrane fluidity and membrane dipole potential treated with bacterial MVs. (A) Fluorescence image showing GUVs composed of DOPC:BSM:Chol in the molar ratio of 5:5:0 doped with 1 mole \% Rhodamine PE (red channel) interaction with DiO labelled bacterial MVs (green channel). Bacterial MVs distribution on equatorial plane of GUVs is represented by merged image of both red and green channels along with the 3D stack of each channel. (B) Fluorescence anisotropy of fully disordered DOPC:BSM:Chol 5:5:0 membrane induced by bacterial MVs, (C) Dipole potential of fully disordered (DOPC:BSM:Chol 5:5:0) treated with increasing concentration of bacterial MVs. (D) Multiple lipid domain can confine the binding of bacterial MVs to partially disordered DOPC:BSM:Chol 4:4:2 membrane (left- Rhodamine PE- (red), centre- DiO (green) and right- merged). Top panel is showing MV binding to equatorial plane of GUVs and the panel below is bacterial MVs 3D-stack of GUV-MVs mixture. (E) Membrane fluidity variation in DOPC:BSM:Chol in molar ratio of 4:4:2 membrane treated with bacterial MVs in increasing concentration. $(F)$ Dipole potential of (DOPC:BSM:Chol) in mole ratio of 4:4:2 treated with bacterial MVs in increasing concentration. (G) Fluorescence image showing GUVs composed of DOPC:BSM:Chol in the molar ratio of 3:3:4 correspond to liquid ordered domain (lo) doped with 1 mole \% Rhodamine PE (red channel) interaction with $\mathrm{DiO}$ labelled MVs (green channel). bacterial MVs has least binding with 
DOPC:BSM:Chol; 3:3:4 represented by merged image of both red and green channels along with the 3D stack of each channel. $(H)$ Changes in anisotropy of DOPC:BSM:Chol (3:3:4) treated with increasing concentration of bacterial MVs. (I) Fluorescence ratio and dipole potential of (DOPC:BSM:Chol 3:3:4) treated with increasing concentration of Bacterial MVs. At least $30 \mathrm{GUVS}$ visualized under microscope and scale bar is $10 \mu \mathrm{M}$. All fluorescence spectroscopic data are shown as mean \pm S.E. from three independent experiments. Significant variations were compared through one-sided ANOVA of membrane condition treated with bacterial MVs with respect to control (only lipid membrane without bacterial MVs), where, $\left({ }^{* * *}\right)$ depicts $p<0.001,\left({ }^{* *}\right) p<0.01$ and $\left({ }^{*}\right) p<0.05$, test data point (membrane treated with bacterial MVs) with no asterisk mark are non-significant. 
Figure 6
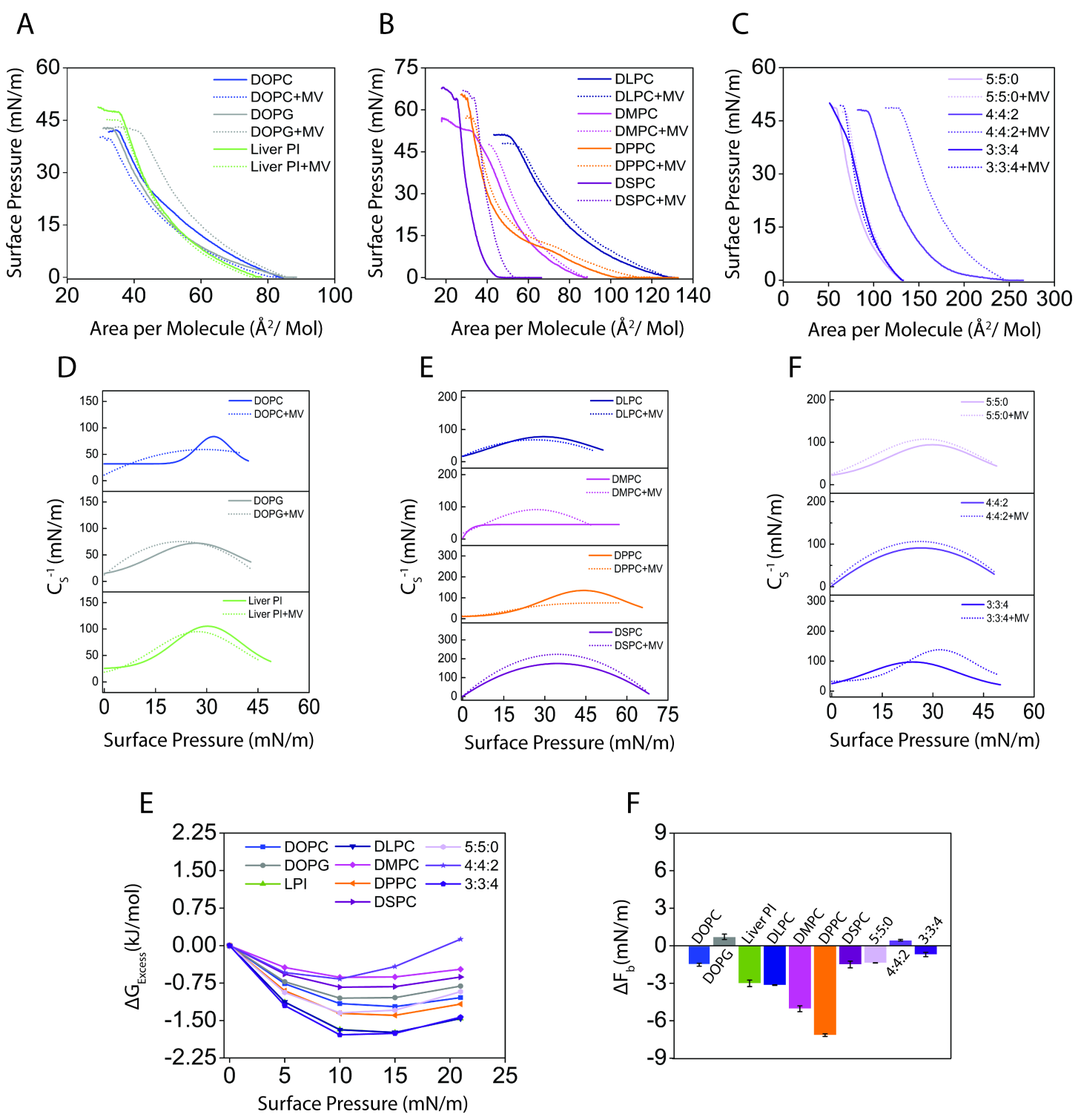

Figure 6. Surface Pressure ( $\pi$ )-mean molecular area (A) isotherms, changes in compressibility modulus, excess free energy change and changes in bending force rigidity of different headgroup containing phospholipid monolayers at air-water interface with and without bacterial MVs in the subphase. T-A isotherms of (A) DOPC, DOPG and Liver PI; (B) DLPC (12:0), DMPC (14:0), DPPC (16:0) and DSPC (18:0); (C) 5:5:0 (BSM:DOPC:cholesterol), 4:4:2 (BSM:DOPC: cholesterol) and 3:3:4 (BSM:DOPC:cholesterol) with and without bacterial MVs obtained at $25^{\circ} \mathrm{C}$. Variability in compressibility modulus plotted as a function of surface pressure acquired for (D) DOPC, DOPG and Liver PI , (E) DLPC (12:0), DMPC (14:0), DPPC (16:0) and DSPC (18:0) and (F) 5:5:0, 4:4:2 and 3:3:4 from respective Langmuir isotherms. (E) Variation of excess Gibb's free energy of mixing of bacterial MVs with lipid monolayer as a function of increasing surface pressures $0 \mathrm{mN} / \mathrm{m}, 5$ $\mathrm{mN} / \mathrm{m}, 10 \mathrm{mN} / \mathrm{m}, 15 \mathrm{mN} / \mathrm{m}$ and $20 \mathrm{mN} / \mathrm{m}$. Bending force $\left(\Delta \mathrm{F}_{\mathrm{b}}\right)$ histogram of DOPC, DOPG, Liver PI, DLPC, DMPC, DPPC, DSPC, 5:5:0, 4:4:2 and 3:3:4 monolayers at the interface in presence of bacterial MVs. All isotherms were recorded with constant barrier compression speed of $8 \mathrm{~mm} / \mathrm{min}$ at $25^{\circ} \mathrm{C}$. All representative isotherms are the mean of independently performed experiments conducted thrice. For 
bioRxiv preprint doi: https://doi.org/10.1101/827519; this version posted February 14,2020 . The copyright holder for this preprint (which was not certified by peer review) is the author/funder. All rights reserved. No reuse allowed without permission.

isotherms and compressibility modulus curves the estimated standard error for data points of surface pressure $(\pi)$ with respect to area per molecule $(A)$ was approximately $\pm 0.05 \mathrm{mN} / \mathrm{m}$. 